\title{
Control of tumor markers using nanotechnology
}

\author{
A. Gómez-Hens, J.M. Fernández-Romero, M.P. Aguilar-Caballos \\ Department of Analytical Chemistry \\ University of Cordoba \\ Annex to "Marie Curie" Building. Campus of Rabanales.14071-Córdoba \\ e-mail:qalgohea@uco.es. Web: http://www.uco.es/FQM-303/
}

Nanotechnology is a current trend in cancer research, more specifically, in cancer diagnosis and therapy. In one hand, nanostructures are useful tools in cell proliferation and tumor marker assays to detect the presence and/or activity of tumor cells at relatively low percentages in biological fluids and/or tissues. On the second, modern therapies are based on drug delivery systems involving the use of nano-carriers, which decrease the side effects to patients and provide a higher effectiveness in the treatment of the disease.

Tumor makers are molecules used as indicators of the presence of cancer, the response to the therapy or the development of the disease. An important group of these markers is constituted by glycoproteins, such as prostate specific antigen (PSA), $\alpha$-fetoprotein (AFP), human chorionic gonadotropin (hCG) and carcinoembryonic antigen (CEA). Other useful markers are the mucinous glycoproteins cancer antigen 125 (CA 125), cancer antigen (CA 15-3) and carbohydrate antigen 19-9 (CA 19-9). These proteins are mainly determined by using immunological mehods, which have traditionally involved the use of enzymes or fluorophores as labels. Nanoparticles (NPs) can be used in tumor marker assays as labels, nanoscaffolds and separation media. Carbon nanotubes, quantum dots, and noble metal, organic polymer, silica and magnetic NPs are the main nanomaterials that have shown their usefulness for these purposes up to date. Generally, the use of nanotechnology in tumor marker assays has provided them with shorter analysis times and higher sensitivity compared to the conventional methods. These assays can be miniaturized using microarray or microfluidic systems and allow the individual or multiplexed determination of tumor markers using direct, competitive or sandwich schemes. 